\title{
Spontaneous coronary artery dissection in the third trimester-Implications for investigation and delivery
}

\author{
Thomas Paxton-Hall $^{1}{ }^{\oplus} \mid$ Pranali Desai $^{2} \mid$ Nicholas Seton $^{1} \mid$ Chris Arthur $^{3}$
}

${ }^{1}$ Gold Coast University Hospital,

Southport, Qld, Australia

${ }^{2}$ Mater Mothers Hospital, Brisbane, Qld, Australia

${ }^{3}$ Griffith University, Southport, Qld, Australia

\section{Correspondence}

Thomas Paxton-Hall, Gold Coast

University Hospital, Southport, Qld, Australia.

Email: paxtonhall.thomas@gmail.com

\begin{abstract}
This case of spontaneous coronary artery dissection in pregnancy highlights the diagnostic, treatment and delivery dilemmas in the antepartum period. This is a rare condition that usually manifests in the postpartum period. This case provides multidisciplinary considerations that were encountered for optimal maternal and fetal outcome.
\end{abstract}

\section{K E Y W O R D S}

Cesarean section, spontaneous coronary artery dissection

Funding information

None

\section{1 | BACKGROUND}

Spontaneous coronary artery dissection (SCAD) is a rare phenomenon, which occurs predominantly in the immediate postpartum period in young women. A SCAD while pregnant is very rare and poses significant risk to the mother and unborn child, and with minimal data on SCAD antenatally, the obstetric dilemma of timing and mode of delivery is not clear. This case of a 33-year-old nulliparous woman with a SCAD at 33 weeks gestation focuses on the diagnostic and delivery implications from a cardiology, anesthetic and obstetric point of view. Given its rarity, this case aims to increase the database of SCAD in pregnancy to provide practice points in delivery.

Cardiovascular disease is present in $1 \%-4 \%$ of pregnancies and is escalating which is considered to be as a result of factors such as advancing maternal age and increasing rate of pregnancy risk factors such as diabetes mellitus, preeclampsia, and multiple gestation pregnancies. Medical advances have resulted in more women with congenital cardiac conditions surviving until, and well past, childbearing age. The relative change in cardiac output and blood volume in pregnancy can alter hemodynamic properties antenatally, intrapartum, and postpartum. While there has been research and development of risk stratification for pregnant women with known cardiac conditions such as valvulopathies and cardiomyopathies, evidence for conditions with intrapartum onset is less established. ${ }^{1,2}$

The incidence of spontaneous coronary artery dissection (SCAD) in pregnancy is a rare phenomenon with management considerations that differ to SCAD in the non-pregnant state or similar. It can be a life-threatening condition which poses delivery considerations if it occurs antenatally. This case report describes a particularly rare case of SCAD in the late pre-term period, in contrast to the immediate postpartum period as is most commonly seen.

\section{CASE}

A 33-year-old nulliparous woman presented to a regional emergency department at 33 weeks' gestation describing subacute chest pain, diaphoresis, and dyspnea. The pregnancy was a spontaneous conception and the antenatal 


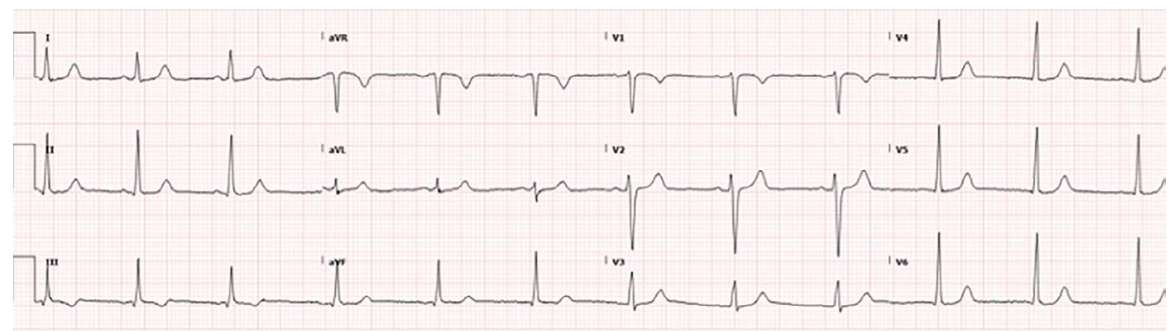

FI G URE 1 ECG on admission with evidence of T-wave inversion in Lead III period had only been complicated by iron deficiency for which the patient was taking oral iron supplements. Her combined first trimester screen and non-invasive prenatal test (NIPT) were low risk, and in addition, she had a normal morphology ultrasound at 20 weeks gestation. Her medical history consisted of recurrent spontaneous pneumothoracies of the left lung in childhood, with the left lung having undergone a pleurodesis. She denied smoking or alcohol use, though admits to historical recreational cocaine use, however, not in the years immediately prior to or during this pregnancy. There was no familial history of cardiac medical conditions.

On presentation to the regional emergency department, she described acute onset, central, dull chest pain with radiation to both axilla, and associated nausea and diaphoresis. This was on the background of two weeks of worsening gastro-esophageal reflux symptoms. There was no associated paraesthesia, pleurisy, or palpitations. The initial workup in the regional emergency department included a chest X-ray and point of care transthoracic echocardiogram (TTE), both of which were normal. An electrocardiogram (ECG) showed equivocal T-wave inversion in Lead III. However, a point of care cardiac troponin went from $0.00 \mathrm{ug} / \mathrm{L}$ to $0.54 \mathrm{ug} / \mathrm{L}$ in eight hours (ref: $0.00-$ $0.04 \mu \mathrm{g} / \mathrm{L}$ ). Given the positive troponin and pregnancy status, she was transferred by road to a tertiary obstetric center. On arrival, a formal troponin was obtained at a level of $4829 \mathrm{ng} / \mathrm{L}$ ( $<10 \mathrm{ng} / \mathrm{L}$ normal). Throughout the event, her observations remained within normal limits. She was admitted for telemetry monitoring in the cardiac care unit (CCU) and was reviewed by the on call obstetric team. After counseling and before further investigation, two doses of $11.4 \mathrm{mg}$ intramuscular betamethasone were administered (with a 24-hour interval) and she was commenced on therapeutic enoxaparin at $1 \mathrm{mg} / \mathrm{kg} /$ day.

Further investigations were conducted including femoral and carotid doppler studies, autoimmune screening, serial ECG, and a formal TTE showing a left ventricular ejection fraction of $68 \%$; all were within normal limits. A computerized tomography pulmonary angiogram (CTPA) and an angiogram of the heart were considered; however, both were abandoned due to the risks of iatrogenic artery dissection and radiation to the fetus. In the absence of an angiogram but with the available results from investigations to date, a SCAD was diagnosed clinically. The diagnosis of a SCAD was made predominantly from the TTE showing normal left ventricular wall function with no wall akinesis, no evidence of infarction on ECG and the significant troponin leak (Figure 1).

During the 7-day acute admission, she remained asymptomatic from a cardiac perspective with normal telemetry. The obstetric team reviewed at least daily, both as a routine and for any maternal pregnancy concerns. Investigations for fetal wellbeing included a Kleihauer test and fetal growth ultrasound, both of which were normal. The estimated fetal weight was on the 40th centile, with a normal amniotic fluid index and doppler studies. A multidisciplinary team (MDT) meeting was undertaken to discuss the antenatal, intrapartum, and postpartum management for this patient. The consensus management was for elective birth at 37 weeks gestation, with either a vaginal birth with a modified second stage or cesarean section considered appropriate, with early regional anesthesia in either event. It was considered that for the intervening weeks, aspirin alone would be suitable anticoagulation, and so the enoxaparin ceased. Given the increased concern regarding SCAD in the postpartum period, a planned 2-week elective admission postpartum in conjunction with telemetry monitoring for the first 48 hours was recommended.

The patient was discharged at 34 weeks gestation on 150mg aspirin daily. She was required to stay within 30 minutes of the hospital and present if she had any concerns. A further MDT with patient involvement was undertaken to plan the mode of delivery. After counseling that the modified second stage would involve an early epidural, telemetry, no active pushing, and a forceps delivery, the patient decided on an elective cesarean section, for which she was consented and planned for at $37+1$ weeks.

An uncomplicated cesarean section was conducted at $37+1$ weeks, resulting in an uncomplicated delivery of a $2.8 \mathrm{~kg}$ baby with APGARS of 7 and 7 under combined spinal-epidural anesthetic. She was commenced on $47.5 \mathrm{mg}$ of extended-release metoprolol and $150 \mathrm{mg}$ of aspirin once daily and remained well for the 2-week admission post-delivery. On discharge, she completed the metoprolol for a further 4 weeks and the aspirin for a total 12 weeks. Due to the high risk of iatrogenic dissection 


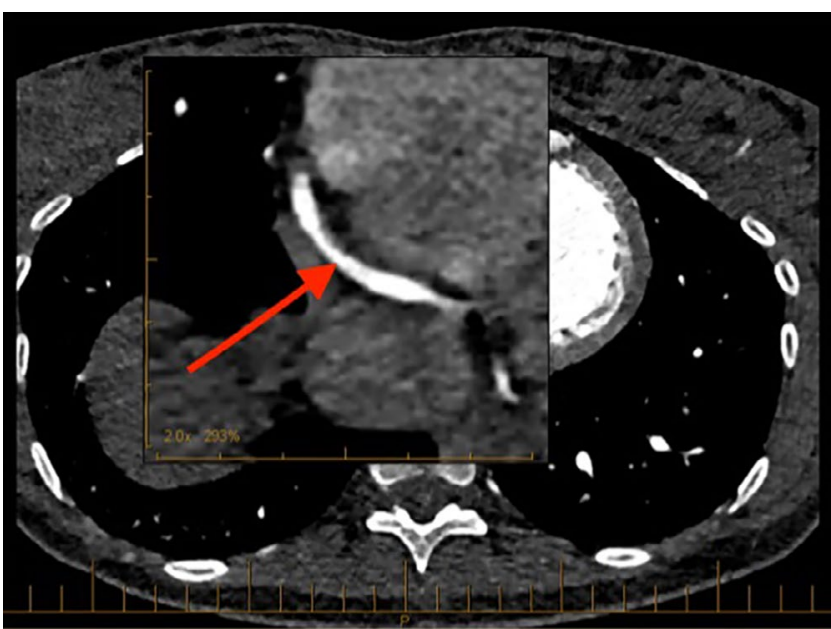

F I G URE 2 CTCA with evident patent right coronary artery

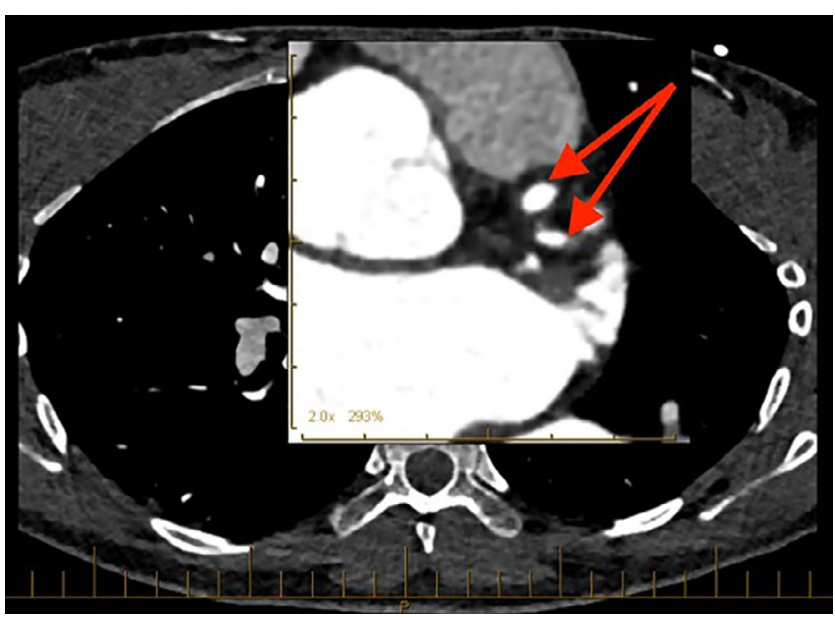

F I G U RE 3 CTCA with evident left coronary and left anterior descending arteries

with an angiogram, a computed tomography coronary angiogram and computed tomography angiogram of the carotid, vertebral, subclavian, renal, and iliac arteries were conducted to exclude aneurysm and resolution of the SCAD. This showed normal vessels and SCAD resolution (no intramural artery hematoma) at 3 months postpartum (Figures 2 and 3).

\section{DISCUSSION}

Chest pain in pregnancy has multiple etiologies with sinister causes ranging from pulmonary embolism, peripartum cardiomyopathy, myocardial infarction, pericarditis, pneumothorax, and SCAD. SCAD is an ischemic event that occurs with an expanding intramural hematoma between the tunica media and intima of an artery resulting in separation of the intima-media complex. This creates a false lumen which in turn compresses the true lumen of the vessel resulting in ischemia and therefore an acute coronary syndrome (ACS). ${ }^{3}$ Among the general population, there is great variation in type of primary presenting ACS being ST-elevated myocardial infarction (STEMI), nonST-elevated myocardial infarction (NSTEMI), and unstable angina. ${ }^{4-6}$ The vast majority of SCAD presentations (87\%-95\%) occur in women at a mean age of 41 years old, slightly lower than the 45 year mean for SCAD in males, yet most of these women do not have typical cardiovascular risk factors. ${ }^{7,8}$ Patients with SCAD are grouped into three subsets: coronary artherosclerotic disease (CAD), females in the peripartum period, and idiopathic SCAD. ${ }^{9}$

SCAD is responsible for $25 \%$ of myocardial infarctions (MI) antenatally and $50 \%$ of MI in the peri- and postpartum periods. ${ }^{10}$ Patients with pregnancy-associated spontaneous coronary artery dissection ( $\mathrm{p}$-SCAD) generally present with a more severe clinical manifestations when compared with SCAD not associated with pregnancy. SCAD antepartum is very rare but is associated with high maternal and fetal morbidity. ${ }^{11,12}$ As per the Mayo SCAD Registry, SCAD in pregnancy is more likely to occur postpartum, most likely to be associated with STEMI, and significantly correlated to multiparity, infertility treatment, and pre-ecclampsia. ${ }^{11,13}$ SCAD can be associated with connective tissue disorders, vasculitis polycystic kidney disease, and use of particular drugs. Its pathophysiology in pregnancy is multifactorial, likely due to a combination of sexual hormones and an increase in shearing force from a hyperdynamic circulation.

For those presenting with typical or atypical symptoms consistent with an ACS, investigation typically starts with standard pathology such as serial troponin levels in addition to serial ECG and echocardiogram. SCAD must be considered among the diagnostic differentials. This is followed by the diagnostic modality of invasive coronary angiography for which there is usually significant concern surrounding fetal radiation. It is estimated that procedural exposure contributes to approximately 1 in 8000 of childhood cancer cases. ${ }^{14}$ Unlike classic atherogenic myocardial infarction, percutaneous intervention (PCI) is not routinely recommended in SCAD. This is mainly due to the unpredictable nature of PCI and the high risk of hematoma propagation along the vessel (occurs in approximately one third of cases). ${ }^{15}$ Other modalities such as intravascular ultrasound and optical coherence tomography are fast becoming beneficial in vessel morphology, however, are typically reserved for cases where the diagnosis is unclear or where intervention of the lesion may be required. ${ }^{15}$

The vast majority of antenatal SCAD cases occur in the postpartum period; therefore, data on delivery planning in the context of antenatal SCAD are very limited. A 
retrospective cohort study of 54 women showed that only 4 were diagnosed with SCAD antenatally; only 1 of these women chose to undergo elective termination, while the other 3 women had emergency cesarean sections, though the indication for cesarean delivery was not reported. ${ }^{16}$ Another large case analysis compiling 120 cases of SCAD in pregnancy in contemporary literature showed that only $17.5 \%$ presented in the 3 rd trimester and $6 \%$ in the 2 nd trimester. $^{16}$

Currently, the preferred treatment for antenatal SCAD is conservative; however, intervention may be required in the circumstances such as ongoing or recurrent clinical or ECG-demonstrated ischemia, cardiogenic shock requiring support, sustained VT or VF, or a left main coronary artery dissection. ${ }^{17}$ There is evidence that suggests any surgical intervention required to stabilize the female patient with a SCAD in pregnancy, such as PCI and coronary artery bypass graft (CABG), are best performed postnatally, and as such if surgical intervention is required then emergent pre-procedure birth of the baby should be considered. ${ }^{16}$ Havakuk et.al. found that of the 4 women who underwent CABG antenatally, $50 \%$ experienced intrauterine fetal loss and the other 50\% experienced significant fetal complications. While there is limited published research on the specifics of delivery planning and intrapartum management of SCAD in pregnancy, it is a known ischemic cardiac event, and therefore, principles applied to other ischemic cardiac conditions in pregnancy can be extrapolated to SCAD in pregnancy. In order to achieve rate control, $B 1$ adrenergic blockers are the preferred treatment option, in addition to being widely used in other pregnancy conditions such as hypertension, arrhythmias, mitral stenosis, and Marfan syndrome. ${ }^{10}$ It is, however, prudent to consider fetal effects such as bradycardia, hypoglycemia, and apnea of the newborn.

It is proposed that the hormonal milieu associated with the pregnant and postpartum state are partially responsible for p-SCAD. In particular, high levels of progesterone may contribute to changes in protein composition, degradation of reticulin, collagen and elastic fibers and hypertrophy of smooth muscle of the vessel wall. ${ }^{18}$ This, in combination with the other cardiac hemodynamic changes of pregnancy, such as increased cardiac output/ intravascular volume and decreased peripheral vascular resistance (which are estrogen mediated), can ultimately increase the risk of rupture of a weakened vessel wall. ${ }^{16}$

It is unclear if the female sex hormones used for contraception increase the risk of SCAD in a similar way to pregnancy, as they are likely to only be only part of the mechanism. However, there are at least 7 published cases of women who experienced SCAD in the context of oral contraceptive pill use, suggesting a possible association. ${ }^{18-20}$ As well as this, other hyperestrogenic states have been associated with SCAD, such as hepatic cirrhosis. ${ }^{21}$ Havakuk et al. showed that when compared to SCAD, women with p-SCAD had a higher risk of persistence and often progression of SCAD. This was demonstrated in one third of their population. ${ }^{16}$ This ongoing fragility of the vessel wall steers the authors to advocate for an individualized discussion which takes into consideration the possible risk of SCAD recurrence with contraceptive use and that associated with repeat pregnancy. Further research into this area would be helpful for this patient cohort.

\section{4 | CONCLUSION}

This case highlights the diagnostic and management dilemmas of SCAD in pregnancy. It can be a difficult clinical scenario to balance patient autonomy and safety, particularly on the background of a small evidence base with limited recommendations for optimal investigations and delivery modality. This case contributes valuable demographic data to the rare condition of SCAD intrapartum. Further research into this unique pregnancy-associated cardiac condition would provide a valuable contribution to the evidence base for clinicians faced with this scenario in future.

\section{ACKNOWLEDGMENTS}

Published with written consent of the patient.

\section{CONFLICTS OF INTEREST}

The authors declare that they have no conflicts of interest.

\section{AUTHOR CONTRIBUTIONS}

Both T.P-H and P.D were the primary authors with N.S proving specialist cardiac review and C.A verified the article as a whole.

\section{ETHICAL APPROVAL}

All authors mentioned in this manuscript agree on authorship and order of authorship.

\section{DATA AVAILABILITY STATEMENT}

No datasets were used in the writing of this case report.

\section{ORCID}

Thomas Paxton-Hall (1) https://orcid. org/0000-0003-2376-3062

\section{REFERENCES}

1. Silversides C, Grewal J, Mason J, et al. Pregnancy Outcomes in Women With Heart Disease: The CARPREG II Study. J Am Coll Cardiol. 2018;71(21):2419-2430. 
2. Greutmann M, Pieper PG. Pregnancy in women with congenital heart disease. Eur Heart J. 2015;36(37):2491-2499.

3. Waterbury T, Tweet M, Hayes S, et al. Early natural history of spontaneous coronary artery dissection. Circ Cardiovasc Interv. 2018;11:e006772.

4. Mortensen K, Thuesen L, Kristensen I, Christiansen E. Spontaneous coronary artery dissection: a Western Denmark Heart Registry Study. Catheter Cardiovascular Intervention. 2009;74:710-717.

5. Tweet M, Hayes S, Pitta S, et al. Clinical features, management, and prognosis of spontaneous coronary artery dissection. Circulation. 2012;126:579-588.

6. Kansara P, Graham S. Spontaneous coronary artery dissection: case series with extended follow up. J Invasive Cardiol. 2011;23:76-80.

7. Shamloo B, Chintala R, Nasur A, et al. Spontaneous coronary artery dissection: aggressive vs. conservative therapy. J Invasive Cardiol. 2010;22:222-228.

8. Kok S, Hayes S, Cutrer F, et al. Prevalence and clinical factors of migraine in patients with spontaneous coronary artery dissection. J Am Heart Assoc. 2018;7:e010140.

9. DeMaio S, Kinsella S, Silverman M. Clinical course and longterm prognosis of spontaneous coronary artery dissection. Am J Cardiol. 1989;64:471-474.

10. Roth A, Elkayam U. Acute myocardial infarction associated with pregnancy. J Am Coll Cardiol. 2008;52:171-180.

11. Tweet M, Hayes S, Codsi E, Gulati R, Rose C, Best P. Spontaneous Coronary Artery Dissection Associated With Pregnancy. J Am Coll Cardiol. 2017;70(4):426-435.

12. Roth A, Elkayam U. Acute myocardial infarction associated with pregnancy-an update. Ann Intern Med. 1996;125:751-776.

13. Saw J, Humphries K, Aymong E, et al. Spontaneous coronary artery dissection: clinical outcomes and risk of reoccurrence. $J$ Am Coll Cardiol. 2017;70(9):1148-1158.

14. Cauldwell M, Baris L, Roos-Hesselink J, Johnson M. Ischaemic heart disease and pregnancy. Heart. 2019;105:189-195.
15. Hayes S, Tweet M, Adlam D, et al. Spontaneous coronary artery dissection JACC State-Of-The-Art Review. J Am Coll Cardiol. 2020;76:961-984.

16. Havakuk O, Goland S, Mehra A, Elkayam U. Pregnancy and the risk of spontaneous coronary artery dissection. Circulation. 2017;10(3):e004941. https://doi.org/10.1161/CIRCINTERV ENTIONS.117.004941

17. Vijayaraghavan R, Verma S, Gupta N, Saw J. Pregnancyrelated spontaneous coronary artery dissection. Circulation. 2014;130(21):1915-1920. https://doi.org/10.1161/CIRCULATIO NAHA.114.011422

18. Evangelou D, Letsas K, Korantzopoulos P, Antonellis I, Sioras E, Kardaras F. Spontaneous coronary artery dissection associated with oral contraceptive use: a case report and review of the literature. Int J Cardiol. 2006;112(3):380-382. https://doi. org/10.1016/j.ijcard.2005.07.069

19. Walters A, Miller K, Badawy I, Martini M. Multivessel spontaneous coronary artery dissection in a young woman on estrogen-progesterone contraceptive patch. J Am Coll Cardiol. 2020;75(11_Supplement_1):3305.

20. Zehir R, Karabay C, Kocabay G. Myocardial infarction and spontaneous dissection of coronary artery due to oral contraceptive. J Cardiovasc Med. 2011;12(6):448-450. https://doi. org/10.2459/JCM.0b013e3283467fc9

21. Patrignani A, Purcaro A, Gabrielli G, Ciampani N. Spontaneous coronary artery dissection and elevated levels of lipoprotein(a) in a young man: a causal association? J Cardiovasc Med. 2011;12(6):446448. https://doi.org/10.2459/JCM.0b013e3283468178

How to cite this article: Paxton-Hall T, Desai $P$, Seton N, Arthur C. Spontaneous coronary artery dissection in the third trimester-Implications for investigation and delivery. Clin Case Rep. 2021;9:e04675. https://doi.org/10.1002/ccr3.4675 